\title{
The Calcium Sensitizer Levosimendan Improves Human Diaphragm Function
}

\author{
Jonne Doorduin1, Christer A. Sinderby,3, Jennifer Beck ${ }^{3,4}$, Dick F. Stegeman ${ }^{5,6}$, Hieronymus W. H. van Hees7, \\ Johannes G. van der Hoeven ${ }^{1}$, and Leo M. A. Heunks ${ }^{1}$
}

\begin{abstract}
${ }^{1}$ Department of Critical Care Medicine, Radboud University Nijmegen Medical Centre, The Netherlands; ${ }^{2}$ Department of Medicine, Division of Critical Care Medicine, University of Toronto; ${ }^{3}$ Keenan Research Centre in the Li Ka Shing Knowledge Institute of St. Michael's Hospital, University of Toronto; ${ }^{4}$ Department of Pediatrics, St. Michael's Hospital, University of Toronto, Toronto, Canada; ${ }^{5}$ Department of Neurology, Radboud University Nijmegen Medical Centre, The Netherlands; ${ }^{6}$ Faculty of Human Movement Sciences, Research Institute MOVE, VU University, Amsterdam, The Netherlands; and ${ }^{7}$ Department of Pulmonary Diseases, Radboud University Nijmegen Medical Centre, Nijmegen, The Netherlands
\end{abstract}

\begin{abstract}
Rationale: Acquired diaphragm muscle weakness is a key feature in several chronic conditions, including chronic obstructive pulmonary disease, congestive heart failure, and difficult weaning from mechanical ventilation. No drugs are available to improve respiratory muscle function in these patients. Recently, we have shown that the calcium sensitizer levosimendan enhances the force-generating capacity of isolated diaphragm fibers.
\end{abstract}

Objectives: To investigate the effects of the calcium sensitizer levosimendan on in vivo human diaphragm function.

Methods: In a double-blind, randomized, crossover design, $\mathbf{3 0}$ healthy subjects performed two identical inspiratory loading tasks. After the first loading task, subjects received levosimendan $(40 \mu \mathrm{g} / \mathrm{kg}$ bolus followed by $0.1 / 0.2 \mu \mathrm{g} / \mathrm{kg} / \mathrm{min}$ continuous infusion) or placebo. Transdiaphragmatic pressure, diaphragm electrical activity, and their relationship (neuromechanical efficiency) were measured during loading. Magnetic phrenic nerve stimulation was performed before the first loading task and after bolus administration to assess twitch contractility. Center frequency of diaphragm electrical activity was evaluated to study the effects of levosimendan on muscle fiber conduction velocity. Measurements and Main Results: The placebo group showed a 9\% $(P=$ $0.01)$ loss of twitch contractility after loaded breathing, whereas no loss in contractility was observed in the levosimendan group. Neuromechanical efficiency of the diaphragm during loading improved by $21 \%(P<0.05)$ in the levosimendan group. Baseline center frequency of diaphragm electrical activity was reduced after levosimendan administration $(P<0.05)$.

Conclusions: The calcium sensitizer levosimendan improves neuromechanical efficiency and contractile function of the human diaphragm. Our findings suggest a new therapeutic approach to improve respiratory muscle function in patients with respiratory failure.

Keywords: diaphragm; muscle weakness; calcium sensitization; levosimendan

Impaired force generation of the respiratory muscles has been recognized in a variety of diseases, including chronic obstructive pulmonary disease (COPD) and congestive heart failure, and in critically ill patients (1-7). The pathophysiological substrate of

(Received in original form July 14, 2011; accepted in final form September 13, 2011) Supported by institutional resources.

Author contributions: Literature search, J.D., L.H.; study design, J.D., C.S., J.B., D.S., L.H.; data collection, J.D., L.H.; data analysis, J.D., L.H.; data interpretation, J.D., C.S., J.B., D.S., H.H., J.H., L.H.; writing, J.D., C.S., J.B., D.S., H.H., J.H., L.H. Correspondence and requests for reprints should be addressed to Leo Heunks, M.D., Ph.D., Radboud University Nijmegen Medical Centre, Department of Critical Care Medicine, Geert Grooteplein zuid 10, 6525 GA Nijmegen, The Netherlands. E-mail: L.Heunks@ic.umcn.nl

This article has an online supplement, which is accessible from this issue's table of contents at www.atsjournals.org

Am J Respir Crit Care Med Vol 185, Iss. 1, pp 90-95, Jan 1, 2012 Copyright $\odot 2012$ by the American Thoracic Society

Originally Published in Press as DOI: 10.1164/rccm.201107-12680C on September 29, 2011 Internet address: www.atsjournals.org

\section{AT A GLANCE COMMENTARY}

Scientific Knowledge on the Subject

Respiratory muscle weakness frequently occurs in chronic diseases and contributes to morbidity and mortality. There is no specific pharmacological treatment available to improve respiratory muscle function in patients with impeding respiratory muscle failure. Previous studies have demonstrated that calcium sensitizers improve in vitro function of the respiratory muscles.

\section{What This Study Adds to the Field}

We report that the calcium sensitizer levosimendan improves neuromechanical efficiency of the human diaphragm. In addition, levosimendan restored the loss of diaphragm contractility after an intervention of loaded breathing. These findings suggest that levosimendan treatment may be an effective strategy to improve muscle function in patients with respiratory muscle weakness.

diaphragm dysfunction in these disorders is multifactorial and includes muscle fiber atrophy and contractile protein dysfunction $(3,8-11)$. We found that diaphragm muscle fibers of patients with COPD display reduced sensitivity of the contractile proteins to calcium $(9,12)$. In other words, more calcium is needed to develop the same amount of force as in the non-COPD diaphragm, resulting in impaired contractile efficiency of the diaphragm muscle. Subsequent studies have shown that calcium sensitivity is also reduced in the diaphragm of animal models of congestive heart failure and prolonged mechanical ventilation $(2,13)$. Despite a better understanding of respiratory muscle dysfunction in chronic diseases, no drug is available to improve respiratory muscle function in humans.

Levosimendan is a clinically used calcium sensitizer that has been approved to enhance cardiac contractility in patients with acute heart failure. Clinical studies have shown that levosimendan improves cardiac function in these patients (14). Levosimendan enhances the binding of calcium to troponin $\mathrm{C}$, thereby improving the responsiveness of myofilaments to calcium. Accordingly, a greater amount of force is generated for the same level of cytosolic calcium, resulting in enhanced contractile efficiency. Levosimendan has vasodilatory properties mediated by the opening of the ATP-sensitive potassium $\left(\mathrm{K}_{\mathrm{ATP}}\right)$ channels (15).

Recently, we have shown that levosimendan enhances calcium sensitivity of permeabilized muscle fibers obtained from the human diaphragm, including patients with COPD (12). However, the effects of calcium sensitizing on the human diaphragm in vivo have not been studied. Based on our in vitro data (12), we 
hypothesize that levosimendan improves contractile function of the human diaphragm in vivo through calcium sensitizing and that levosimendan would decrease muscle fiber conduction velocity of the diaphragm through its effects on $\mathrm{K}_{\mathrm{ATP}}$ channels in diaphragm fibers. We tested this hypothesis in a double-blind, placebo-controlled, crossover design in healthy subjects performing inspiratory loading tasks. Some of the results of this study have been previously reported in the form of an abstract (16).

\section{METHODS}

We enrolled 30 healthy volunteers in this trial. The protocol was approved by the ethical committee of the Radboud University Nijmegen Medical Centre and registered at ClinicalTrials.gov (NCT01101620). All subjects gave their informed consent.

\section{Esophageal Catheter}

Diaphragm electromyographic activity (EMGdi), esophageal pressure (Pes), and gastric pressure (Pga) were obtained with a multielectrode esophageal catheter with two balloons (details are provided in the online supplement). Transdiaphragmatic pressure (Pdi) was calculated as Pga - Pes.

\section{Magnetic Stimulation and Maximal Inspiratory Effort}

Cervical magnetic stimulation of the phrenic nerves was performed to measure twitch Pdi $\left(\mathrm{Pdi}_{\mathrm{tw}}\right)$ and compound muscle action potential of the diaphragm (CMAPdi). Details about the stimulation protocol are provided in the online supplement.

Maximal voluntary Pdi $\left(\mathrm{Pdi}_{\max }\right)$ was measured as mean Pdi in the first second during a maximal inspiratory effort against a closed valve at functional residual capacity.

\section{Inspiratory Loading Task}

Each subject performed two identical loading tasks, one before and one after administration of the study medication. Sitting in upright position with uncast abdomen, subjects breathed through a mouthpiece while wearing a nose clip. Subjects performed intermittent inspiratory maneuvers of 10 seconds against a closed valve (near-isometric contractions) followed by 7 seconds of unloaded breathing. Duty cycle was imposed by a sound signal, and subjects were asked to target $40 \%$ of Pdi $\mathrm{imax}_{\text {. Visual }}$ feedback of Pdi was provided. Total loading task duration was 10 minutes. During loading, EMGdi and Pdi were recorded continuously, as well as in unloaded conditions approximately 5 minutes before and after loading. Respiratory effort sensation was scored with a Borg scale (range, 6-20) at 1, 3, 6, and 9 minutes into loading.

\section{Experimental Protocol}

The protocol is presented in Figure 1. $\mathrm{Pdi}_{\mathrm{tw}}$ and $\mathrm{Pdi}_{\max }$ were measured and followed by the first loading task and 30 minutes of unloaded breathing. After randomization, subjects received levosimendan bolus $(40 \mu \mathrm{g} / \mathrm{kg}$ bodyweight, intravenously) or an equal volume of placebo in 10 minutes. $\mathrm{Pdi}_{\mathrm{tw}}$ and $\mathrm{Pdi}_{\max }$ measurements were repeated and followed by 30 minutes of continuous levosimendan $(0.1 \mu \mathrm{g} / \mathrm{kg}$ bodyweight $/ \mathrm{min}$, intravenously) or placebo infusion. A second loading task was performed while infusing levosimendan $(0.2 \mu \mathrm{g} / \mathrm{kg} / \mathrm{min}$, intravenously) or placebo.

Heart rate, end tidal carbon dioxide $\left(\right.$ et $\left.\mathrm{CO}_{2}\right)$, and peripheral oxygen saturation $\left(\mathrm{Sp}_{\mathrm{O}_{2}}\right)$ were monitored continuously. Blood pressure was measured noninvasively every 10 minutes and during bolus administration each minute. In six subjects, cardiac output was determined by transthoracic echocardiography directly before and after bolus administration.

\section{Data Analysis and Statistics}

Measurement variables were analyzed offline in Matlab R2009b (The Mathworks, Natick, MA) (details are provided in the online supplement). The ratio of mean inspiratory Pdi and EMGdi amplitude was calculated as a measure of the neuromechanical efficiency of the

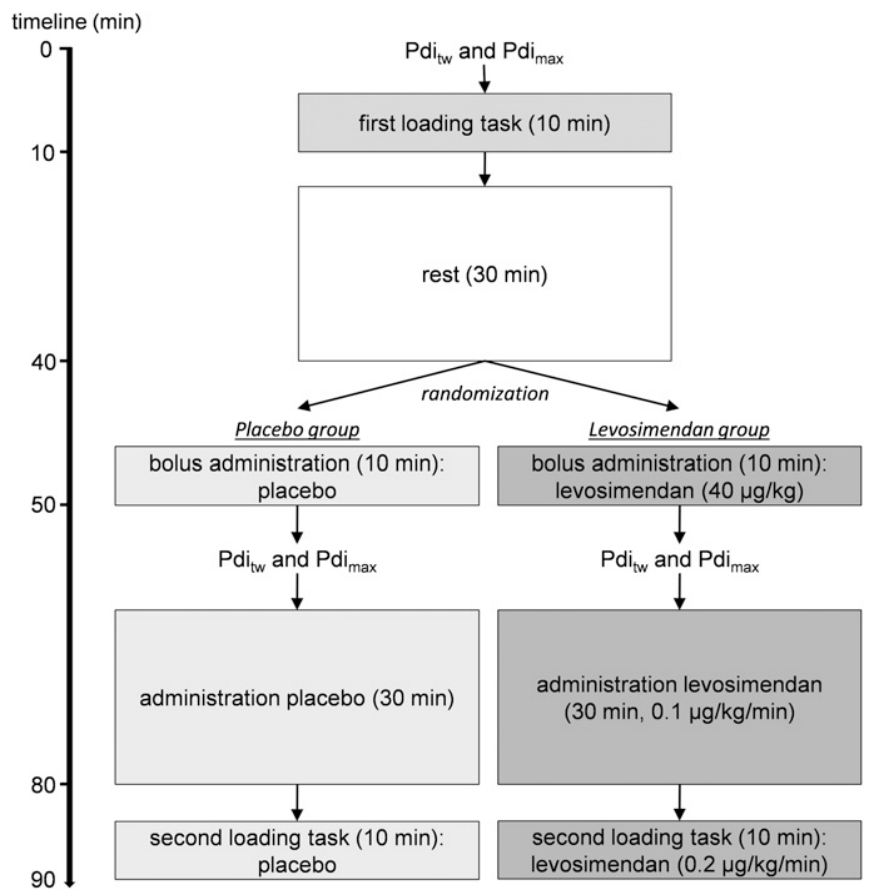

Figure 1. Schematic description of the protocol. Pdi $_{\max }=$ maximum transdiaphragmatic pressure; $\mathrm{Pdi}_{\mathrm{tw}}=$ twitch transdiaphragmatic pressure.

diaphragm. Changes in muscle fiber conduction velocity were evaluated using the power spectrum center frequency (CFdi) of the EMGdi (details are provided in the online supplement). Comparisons were made with the appropriate $t$ test. Changes over time during loaded breathing were analyzed using repeated measures ANOVA. Values are means \pm SEM, and $P<0.05$ was considered significant. Statistical analyses were performed with SPSS 16.0 (SPSS, Chicago, IL).

\section{RESULTS}

Baseline characteristics of the subjects (male/female, 23/7; age, $22 \pm 0.4 \mathrm{yr}$; bodyweight, $74 \pm 1 \mathrm{~kg}$; and body mass index, $23 \pm$ $0.4 \mathrm{~kg} / \mathrm{m}^{2}$ ) were not different between the placebo and levosimendan groups. All subjects $(\mathrm{n}=30)$ received study medication as dictated by the protocol. One subject was excluded due to technical problems with the esophageal catheter. Two subjects were not able to maintain targeted Pdi $\left(<30 \%\right.$ of $\left.\mathrm{Pdi}_{\max }\right)$ during the first loading task and were excluded from data analysis. The remaining subjects (placebo, $\mathrm{n}=13$; levosimendan, $\mathrm{n}=14$ ) were able to keep their targeted Pdi during both loading tasks $(44 \pm 1 \%$ and $43 \pm 2 \%)$, indicating compliance to the protocol. $\mathrm{Sp}_{\mathrm{O}_{2}}$ was above $96 \%$ in all subjects during the study; no supplemental oxygen was given. There were no differences in etCO between the placebo and levosimendan group during loaded breathing.

Furthermore, there were no serious adverse events during the experimental protocol. One subject, receiving levosimendan, experienced a mild degree of nausea after completion of the study.

\section{Diaphragm Contractility}

Cervical magnetic stimulation of the phrenic nerves resulted in reproducible twitch pressures in all subjects. Data from magnetic stimulation and maximal inspiratory maneuvers, as well as hemodynamic data, are given in Table 1 . There was no significant difference in $\mathrm{Pdi}_{\text {tw }}$ and $\mathrm{Pdi}_{\text {max }}$ between the placebo and the levosimendan groups before study medication.

Figure 2 shows a decreased contractile response of the diaphragm after the first loading task in a subject receiving placebo. 
TABLE 1. DIAPHRAGM CONTRACTILITY AND HEMODYNAMIC VARIABLES AT BASELINE AND AFTER LOADING + BOLUS ADMINISTRATION

\begin{tabular}{|c|c|c|c|c|}
\hline \multirow[b]{2}{*}{ Variables } & \multicolumn{2}{|r|}{ Placebo $(n=13)$} & \multicolumn{2}{|c|}{ Levosimendan $(n=14)$} \\
\hline & Baseline & After Loading + Bolus Administration & Baseline & After Loading + Bolus Administration \\
\hline \multicolumn{5}{|c|}{ Diaphragm contractility* } \\
\hline $\mathrm{Pdi}_{\mathrm{tw}}, \mathrm{cm} \mathrm{H} \mathrm{H}_{2} \mathrm{O}$ & $35 \pm 2^{\dagger}$ & $32 \pm 2^{\ddagger}$ & $30 \pm 2$ & $31 \pm 2$ \\
\hline CMAPdi, mV & $1.3 \pm 0.2$ & $1.3 \pm 0.2$ & $1.0 \pm 0.1$ & $1.1 \pm 0.1$ \\
\hline $\mathrm{Pdi}_{\max }, \mathrm{cm} \mathrm{H}_{2} \mathrm{O}$ & $130 \pm 8$ & $122 \pm 7^{\ddagger}$ & $123 \pm 11$ & $120 \pm 8$ \\
\hline $\mathrm{EMGdi}_{\max } \mu \mathrm{V}$ & $76 \pm 9$ & $81 \pm 10$ & $77 \pm 8$ & $74 \pm 10$ \\
\hline \multicolumn{5}{|c|}{ Hemodynamic variables $^{\S}$} \\
\hline MAP, mm Hg & $92 \pm 2$ & $89 \pm 4$ & $90 \pm 3$ & $86 \pm 4$ \\
\hline$H R, b p m$ & $71 \pm 3$ & $73 \pm 3$ & $68 \pm 2$ & $83 \pm 3^{\ddagger}$ \\
\hline $\mathrm{CO}, \mathrm{L} / \mathrm{min}$ & $4.5 \pm 1.3$ & $4.6 \pm 0.4$ & $6.2 \pm 0.7$ & $7.0 \pm 0.7^{\ddagger}$ \\
\hline
\end{tabular}

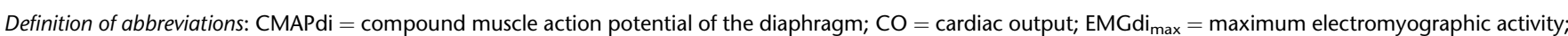
$\mathrm{MAP}=$ mean arterial pressure; $\mathrm{Pdi}_{\max }=$ maximum transdiaphragmatic pressure; $\mathrm{Pdi}_{\mathrm{tw}}=$ twitch transdiaphragmatic pressure; $\mathrm{HR}=$ heart rate

${ }^{*} \mathrm{Pdi}_{\mathrm{tw}}, \mathrm{CMAPdi}, \mathrm{Pdi}_{\max }$ and $\mathrm{EMGdi}_{\max }$ were measured before the first loading task and after bolus administration of study medication.

${ }^{\dagger}$ Data are presented as mean \pm SEM.

‡ Significantly different from before study medication $(P<0.05)$.

$\S \mathrm{MAP}, \mathrm{HR}$, and CO were measured directly before and after bolus administration. CO was measured only in $\mathrm{n}=2$ (placebo group) and $\mathrm{n}=4$ (levosimendan group).

On average, loaded breathing in subjects receiving placebo resulted in significant reductions in $\mathrm{Pdi}_{\mathrm{tw}}(-9 \pm 3 \% ; P=0.01)$ and $\mathrm{Pdi}_{\max }(-5 \pm 3 \% ; P<0.05)$. The group receiving levosimendan after the first period of loaded breathing revealed no significant decrease in $\mathrm{Pdi}_{\mathrm{tw}}$ and $\mathrm{Pdi}_{\max }$ (Figure 3; Table 1).

\section{Neuromechanical Efficiency of the Diaphragm}

In Figure 4, representative tracings of EMGdi and Pdi are presented for the first loading task (before study medication) and the second loaded task (with study medication) for both groups. Neuromechanical efficiency of the diaphragm (Pdi/EMGdi) in the first loading task was not different between the placebo and the levosimendan groups (both $1.3 \pm 0.2 \mathrm{~cm} \mathrm{H}_{2} \mathrm{O} / \mu \mathrm{V}$ ) and remained stable throughout the loading task (Figure 5). Neuromechanical efficiency during unloaded breathing and during the second loading task was improved by $21 \%(P<0.05$, compared with the first loading task) in the levosimendan group, whereas neuromechanical efficiency was not affected in the placebo group (Figure 5). The improved neuromechanical efficiency in subjects receiving levosimendan sustained throughout the entire loading task.

\section{Center Frequency of Diaphragm Electrical Activity}

During both loaded breathing protocols, CFdi decreased over time $(P<0.001)$ (see Figure E1 in the online supplement) in both groups. Administration of levosimendan resulted in a downward shift in baseline CFdi during the second loaded breathing protocol $(P<0.05$; Figure E1), whereas placebo did not affect CFdi.

\section{Respiratory Effort Sensation}

Respiratory effort sensation increased over time $(P<0.001)$ during loaded breathing until a score of 16 was reached. Neither levosimendan nor placebo affected respiratory effort sensation.

\section{DISCUSSION}

The present study is the first to evaluate the effect of the calcium sensitizer levosimendan on human diaphragm function in vivo. In this double-blind, randomized study, an intervention of loaded breathing resulted in significant loss of diaphragm contractility in the placebo group but not in the levosimendan group, suggesting that levosimendan restored diaphragm contractility. Loaded breathing after drug administration was associated with improved neuromechanical efficiency of the diaphragm in the levosimendan group. Furthermore, levosimendan reduced baseline center frequency of diaphragm electrical activity. Levosimendan was well tolerated with negligible side effects.

\section{Calcium Sensitivity and Muscle Fiber Conduction Velocity: Effects of Levosimendan}

Skeletal muscle force develops as intracellular calcium rises and binds to troponin $\mathrm{C}$, resulting in conformational changes in the troponin complex, allowing interaction between actin and myosin to form force-generating cross-bridges. Reuptake of calcium from the cytoplasm into the sarcoplasmic reticulum is a high energyconsuming process (up to $40 \%$ of total energy expenditure). Thus, reduced sensitivity of the troponin complex for calcium requires higher levels of cytoplasmic calcium to generate the same amount of force, requiring higher energy consumption and elevated $\mathrm{CO}_{2}$ production.

Levosimendan belongs to a relatively new class of drugs, the calcium sensitizers and is approved in more than 40 countries worldwide to improve cardiac function in patients with acute heart failure (14). Although the loading dose of levosimendan in this study is higher than recommended for the treatment of

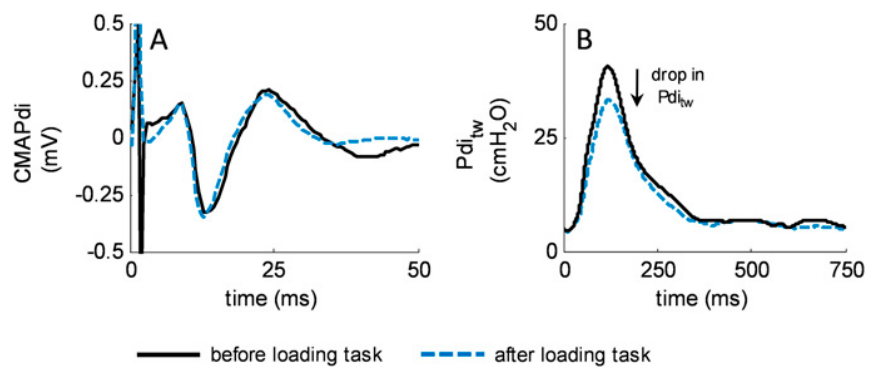

Figure 2. (A) The compound muscle action potential of the diaphragm (CMAPdi) and (B) twitch transdiaphragmatic pressure ( $\mathrm{Pdi}_{\mathrm{tw}}$ ) elicited by cervical magnetic stimulation before (black line) and after the first loading task (blue striped line) in a subject receiving placebo. In response to equal diaphragm activation (i.e., equal CMAPdi), there is decreased contractility after loaded breathing. Note the difference in scale of the $x$ axis between panels $A$ and $B$. 


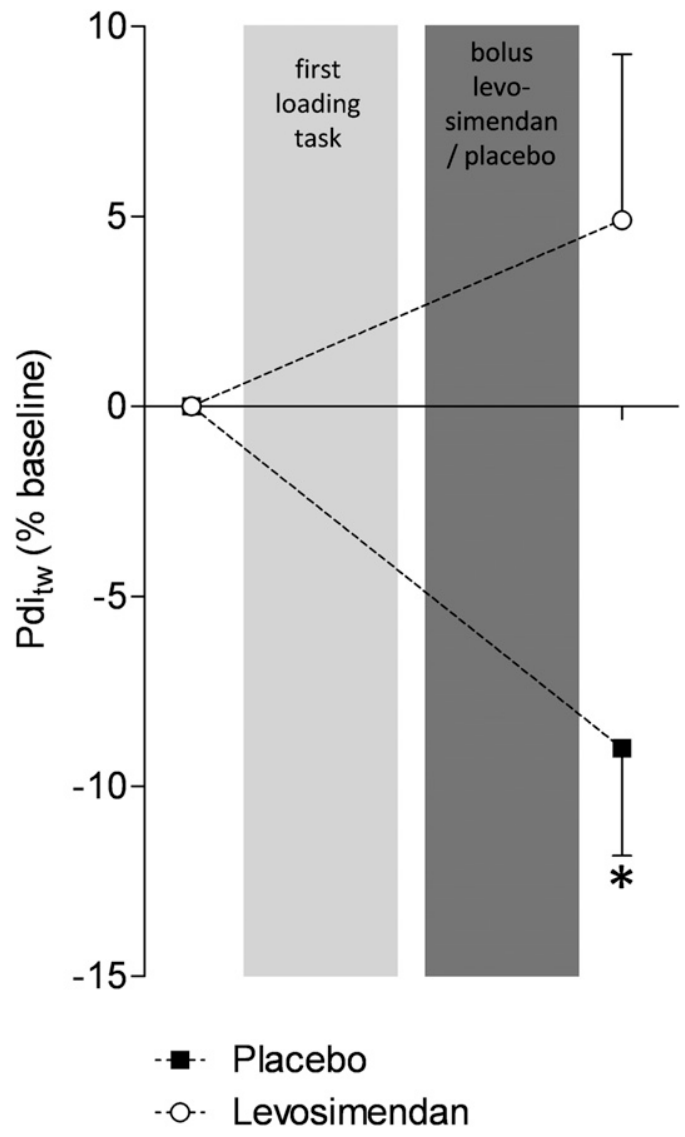

Figure 3. Percentage change in transdiaphragmatic twitch pressure $\left(\mathrm{Pdi}_{\mathrm{tw}}\right)$ from baseline after loading + bolus administration in the placebo (closed squares) and levosimendan (open circles) group. Data are presented as mean \pm SEM (placebo, $n=13$; levosimendan, $n=14$ ). *Significantly different from baseline $(P=0.01)$ and levosimendan group after loading + bolus administration $(P=0.01)$.

acute heart failure (14), the total dose of levosimendan administered is lower than used in clinical practice.

Calcium sensitization by levosimendan occurs through stabilization of the interaction between calcium and the troponin complex during muscle activation (17). Recently, we have shown that levosimendan improves in vitro calcium sensitivity of human diaphragm (skeletal) muscle fibers as well $(12,18)$. Improvement in calcium sensitivity will affect contractile efficiency of the diaphragm (19). In the current study, loaded breathing reduced $\mathrm{Pdi}_{\mathrm{tw}}$ in placebo-treated subjects (Table 1; Figure 3), which is consistent with previous data by Laghi and colleagues (20). However, levosimendan reversed the development of fatigue; $\mathrm{Pdi}_{\mathrm{tw}}$ after loading in these subjects was not different from baseline. Neuromechanical efficiency of the diaphragm during loaded breathing, expressed as Pdi/EMGdi ratio, was enhanced by $21 \%$ after administration of levosimendan (Figure 5). A $21 \%$ improvement in the ability to generate inspiratory pressure for a given neural respiratory effort should be considered a clinically relevant improvement in contractility. In vitro studies have shown that impaired calcium sensitivity of force generation develops during muscle loading and may contribute to contractile failure (21). This is in line with the data from the current study that show that levosimendan reverses the effects of diaphragm force loss (Table 1; Figure 3).

Administration of levosimendan resulted in a downward shift of the CFdi curve during loaded breathing (Figure E1). The decrease in CFdi over time indicates the development of reduced muscle fiber conduction velocity (see the online supplement for details). Reduced muscle fiber conduction velocity is an increased difficulty of muscle fiber action potentials to propagate along the sarcolemma and into $\mathrm{T}$ tubuli (22). Therefore, the downward shift in baseline CFdi in subjects receiving levosimendan most likely reflects a baseline decrease in muscle fiber conduction velocity. These data are in line with the established effects of levosimendan on $\mathrm{K}_{\mathrm{ATP}}$ channel activation (15) and with the finding that extracellular $\left[\mathrm{K}^{+}\right]$accumulation contributes to reduced muscle fiber conduction velocity $(23,24)$. Because administration of levosimendan improves neuromechanical efficiency of the diaphragm by $21 \%$, it is unlikely that extracellular $\left[\mathrm{K}^{+}\right]$accumulation results in functional impairment of the diaphragm. Moreover, preserving a hyperpolarized sarcolemma by activating $\mathrm{K}_{\mathrm{ATP}}$ channels might play a myoprotective role under conditions of metabolic stress (25).

Enhancing calcium sensitivity with levosimendan appears to be a rational and effective approach to improve respiratory muscle function, in particular in patients with imminent respiratory failure.

\section{Clinical Implications}

Reduced force generation of the respiratory muscles has been demonstrated in numerous disorders, including COPD, congestive heart failure, pulmonary hypertension, and patients on mechanical ventilation (1-7). There are no pharmacological interventions available to improve respiratory muscle function.

Aubier and colleagues have previously shown that dopamine (26) and aminophylline (27) improve contractility of the human diaphragm, although the effects of aminophylline are controversial (28). Neither dopamine nor aminophylline has been used in clinical practice to optimize respiratory muscle function, probably due to the small therapeutic window, the risk of severe side effects (aminophylline, dopamine), and the very short half-life (dopamine). Instead, the active levosimendan metabolite OR-1896 has a half-life of 70 to 80 hours, resulting in clinical effect up to 1 week after 24-hour infusion (29). More importantly, both aminophylline and dopamine improve contractility by elevating intracellular calcium concentration, resulting in an increased ATP utilization. As discussed elsewhere (12), inorganic phosphate accumulation contributes to the development of muscle fatigue and depresses calcium sensitivity (30). In addition, elevated energy expenditure increases $\mathrm{CO}_{2}$ production, requiring a higher level of ventilation. This limits the clinical utility of these drugs for the improvement of respiratory muscle function in patients with imminent ventilatory failure. Rather, calcium sensitizers improve muscle contractility without elevating energy expenditure (31). In addition, more efficient breathing may hasten liberation from mechanical ventilation (32). This may be important for patients with respiratory failure, such as acute exacerbation of COPD. Of note, our previous in vitro work showed that the effect of levosimendan is more profound in type- 1 diaphragm fibers than in type-2 fibers (12). Because a fiber type shift toward type- 1 fibers is known to occur in the diaphragm of patients with COPD (8) and congestive heart failure (33), levosimendan treatment can potentially be more effective in these patients than in healthy subjects. Accordingly, the rationale for evaluating the effect of levosimendan on respiratory muscle function in patients with respiratory failure appears highly appropriate.

\section{Experimental Model}

The contractile performance of the diaphragm was evaluated using magnetic stimulation and maximal inspiratory efforts. 

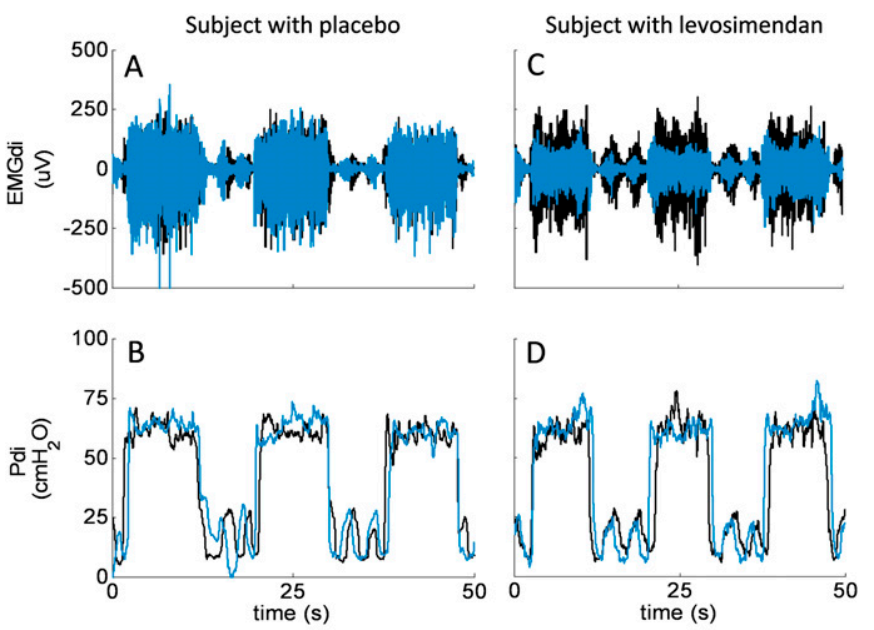

first loading task
(before study medication)

second loading task (with study medication)

Figure 4. Diaphragm electromyography (EMGdi) and transdiaphragmatic pressure (Pdi) during the first (black) and second loading task (blue) in a subject receiving placebo $(A$ and $B)$ and levosimendan ( $C$ and $D$ ). Subjects were asked to target $40 \%$ of $\mathrm{Pdi}_{\text {max }}$. In the subject receiving placebo, there is no change in EMGdi (i.e., neural activation), whereas the subject receiving levosimendan shows decreased neural activation and thus increased neuromechanical efficiency of the diaphragm.

Magnetic stimulation has a clear benefit over voluntary maneuvers because it is effort independent and highly reproducible (34). Values for $\mathrm{Pdi}_{\mathrm{tw}}$ and $\mathrm{Pdi}_{\text {max }}$ reported in our study are similar o those reported by other groups for healthy subjects $(20,35)$. Due to coincidence, baseline $\mathrm{Pdi}_{\mathrm{tw}}$ and CMAPdi amplitude were lower (though insignificant) in the levosimendan group (Table 1), suggesting these subjects were more difficult to stimulate. Correcting for CMAPdi amplitude abolished the baseline difference between the groups (data not shown).

To further evaluate the function of the diaphragm, we measured neuromechanical efficiency of the diaphragm (Pdi/EMGdi) during inspiratory loaded breathing. We found that diaphragm neuromechanical efficiency at $40 \%$ of $\mathrm{Pdi}_{\max }$ was constant over time and that CFdi decreased over time during loaded breathing, which are in line with previous data from Sinderby and colleagues (36). We also found that, despite evidence of twitch force loss, the diaphragm neuromechanical efficiency was not reduced in the placebo group during the second loaded breathing task. Thus, the reductions in maximal contractility were not reflected in the neuromechanical efficiency during submaximal contractions. Regardless of the reason for this inconsistency, the double-blinded randomized design of the study should ensure that the finding of improved neuromechanical efficiency, which occurred only in the levosimendan group, was not due to bias, carry-over effects, or other subjective influences.

For an accurate physiological measurement of EMGdi and CFdi during voluntary breathing, it is necessary to control for changes in muscle-to-electrode distance, electrode positioning with respect to the muscle fiber direction and location, influence of cross-talk from other muscles (including the heart and the esophagus), and electrode movement-induced artifacts. The technology used to measure and process the EMGdi and CFdi in our study minimizes these influences $(37,38)$.

It could be postulated that improved contractile function of the diaphragm resulted from the cardiac inotropic effects of levosimendan. However, subjects in the present study did not have a medical history of cardiac disease, and the effect of levosimendan on cardiac output was modest. Development of $\mathrm{Pdi}_{\mathrm{tw}}$ occurs through a sharp anaerobic maneuver of the diaphragm; therefore, it is unlikely that an improved cardiac output (and oxygen delivery) would explain the restored diaphragm contractility with levosimendan. Moreover, levosimendan improved efficiency already during unloaded breathing and immediately at the start of the loading task, which is unlikely to be the result of improved oxygen delivery to the diaphragm

The dosage of levosimendan used in the current study was derived from earlier studies in healthy subjects that demonstrated limited side effects (39). This is in line with our study. Future studies should evaluate the effects of lower doses of levosimendan on respiratory muscle function in humans.

In conclusion, the present study demonstrates that the calcium sensitizer levosimendan improves contractile function and neuromechanical efficiency of the human diaphragm. These findings suggest a new therapeutic approach for patients with acute respiratory muscle dysfunction.

Author disclosures are available with the text of this article at www.atsjournals.org.

Acknowledgment: The authors thank Dr. M. Kivikko and Prof. P. Rytila from Orion Pharma, Espoo, Finland, for providing levosimendan and placebo; N. Comtois from the Department of Medicine, Division of Critical Care Medicine, St. Michael's Hospital, University of Toronto, Canada and Dr. H. van Dijk from the Department of Neurology, Radboud University Nijmegen Medical Centre, for technical assistance; J. van den Brule from the Department of Critical Care Medicine, Radboud University Nijmegen Medical Centre for performing the transthoracic echocardiography; and the research nurses from the Department of Critical Care Medicine, Radboud University Nijmegen Medical Centre for assistance during the experiments.
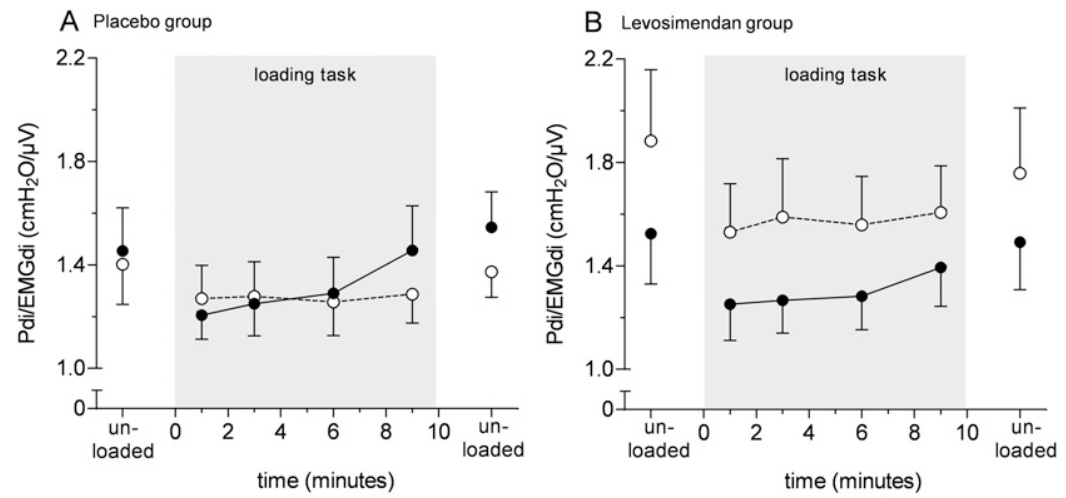

Figure 5. Neuromechanical efficiency of the diaphragm (Pdi/EMGdi) before, during (at 1, 3, 6, and $9 \mathrm{~min}$ ) and after the first and second loading task in the placebo $(A)$ and levosimendan $(B)$ groups. Administration of levosimendan resulted in an increase in diaphragm efficiency $(P<0.05)$ during loaded and unloaded breathing, whereas placebo had no effect on diaphragm efficiency. Data are presented as mean \pm SEM (placebo, $n=13$; levosimendan, $n=14$ ). 


\section{References}

1. Hermans G, Agten A, Testelmans D, Decramer M, Gayan-Ramirez G. Increased duration of mechanical ventilation is associated with decreased diaphragmatic force: a prospective observational study. Crit Care 2010;14:R127.

2. van Hees HW, van der Heijden HF, Ottenheijm CA, Heunks LM, Pigmans CJ, Verheugt FW, Brouwer RM, Dekhuijzen PN. Diaphragm single-fiber weakness and loss of myosin in congestive heart failure rats. Am J Physiol Heart Circ Physiol 2007;293:H819-H828.

3. Ottenheijm CA, Heunks LM, Dekhuijzen PN. Diaphragm muscle fiber dysfunction in chronic obstructive pulmonary disease: toward a pathophysiological concept. Am J Respir Crit Care Med 2007;175:1233-1240.

4. Laghi F, Tobin MJ. Disorders of the respiratory muscles. Am J Respir Crit Care Med 2003;168:10-48.

5. Laghi F, Cattapan SE, Jubran A, Parthasarathy S, Warshawsky P, Choi YS, Tobin MJ. Is weaning failure caused by low-frequency fatigue of the diaphragm? Am J Respir Crit Care Med 2003;167:120-127.

6. Watson AC, Hughes PD, Louise HM, Hart N, Ware RJ, Wendon J, Green M, Moxham J. Measurement of twitch transdiaphragmatic, esophageal, and endotracheal tube pressure with bilateral anterolateral magnetic phrenic nerve stimulation in patients in the intensive care unit. Crit Care Med 2001;29:1325-1331.

7. Hughes PD, Polkey MI, Harrus ML, Coats AJ, Moxham J, Green M. Diaphragm strength in chronic heart failure. Am J Respir Crit Care Med 1999;160:529-534.

8. Levine S, Kaiser L, Leferovich J, Tikunov B. Cellular adaptations in the diaphragm in chronic obstructive pulmonary disease. $N$ Engl J Med 1997;337:1799-1806.

9. Ottenheijm CA, Heunks LM, Sieck GC, Zhan WZ, Jansen SM, Degens H. de Boo T, Dekhuijzen PN. Diaphragm dysfunction in chronic obstructive pulmonary disease. Am J Respir Crit Care Med 2005;172: 200-205.

10. Ottenheijm CA, Heunks LM, Li YP, Jin B, Minnaard R, van Hees HW, Dekhuijzen PN. Activation of the ubiquitin-proteasome pathway in the diaphragm in chronic obstructive pulmonary disease. Am J Respir Crit Care Med 2006;174:997-1002.

11. Levine S, Nguyen T, Taylor N, Friscia ME, Budak MT, Rothenberg P, Zhu J, Sachdeva R, Sonnad S, Kaiser LR, et al. Rapid disuse atrophy of diaphragm fibers in mechanically ventilated humans. $N$ Engl J Med 2008;358:1327-1335.

12. van Hees HW, Dekhuijzen PN, Heunks LM. Levosimendan enhances force generation of diaphragm muscle from patients with chronic obstructive pulmonary disease. Am J Respir Crit Care Med 2009;179:41-47.

13. Ochala J, Radell PJ, Eriksson LI, Larsson L. EMD 57033 partially reverses ventilator-induced diaphragm muscle fibre calcium desensitisation. Pflugers Arch 2010;459:475-483.

14. Follath F, Cleland JG, Just H, Papp JG, Scholz H, Peuhkurinen K, Harjola VP, Mitrovic V, Abdalla M, Sandell EP, et al. Efficacy and safety of intravenous levosimendan compared with dobutamine in severe low-output heart failure (the LIDO study): a randomised double-blind trial. Lancet 2002;360:196-202.

15. Yokoshiki H, Katsube Y, Sunagawa M, Sperelakis N. Levosimendan, a novel $\mathrm{Ca} 2+$ sensitizer, activates the glibenclamide-sensitive $\mathrm{K}+$ channel in rat arterial myocytes. Eur J Pharmacol 1997;333:249-259.

16. Doorduin J, Sinderby CA, van der Hoeven JG, Heunks LM. The calcium sensitizer levosimendan improves contractility and neuromechanical coupling of the diaphragm in healthy subjects [abstract]. Am J Respir Crit Care Med 2011;183:A4244.

17. Sorsa T, Pollesello P, Solaro RJ. The contractile apparatus as a target for drugs against heart failure: interaction of levosimendan, a calcium sensitiser, with cardiac troponin c. Mol Cell Biochem 2004;266: 87-107.

18. van Hees HW, Andrade AG, Linkels M, Dekhuijzen PN, Heunks LM. Levosimendan improves calcium sensitivity of diaphragm muscle fibres from a rat model of heart failure. Br J Pharmacol 2011;162:566573.

19. MacGowan GA. What is the in vivo mechanism of action of levosimendan? J Card Fail 2005;11:482-483.

20. Laghi F, Topeli A, Tobin MJ. Does resistive loading decrease diaphragmatic contractility before task failure? J Appl Physiol 1998;85: 1103-1112.

21. Westerblad H, Allen DG. Changes of myoplasmic calcium concentration during fatigue in single mouse muscle fibers. J Gen Physiol 1991;98: 615-635.

22. Allen DG, Lamb GD, Westerblad H. Skeletal muscle fatigue: cellular mechanisms. Physiol Rev 2008;88:287-332.

23. Fortune E, Lowery MM. Effect of extracellular potassium accumulation on muscle fiber conduction velocity: a simulation study. Ann Biomed Eng 2009;37:2105-2117.

24. Wickenden AD, Prior H, Kelly E, Russell K, Poucher SM, Kumar P. The effects of pharmacological modulation of KATP on the guinea-pig isolated diaphragm. Eur J Pharmacol 1996;302:79-88.

25. Flagg TP, Enkvetchakul D, Koster JC, Nichols CG. Muscle KATP channels: recent insights to energy sensing and myoprotection. Physiol Rev 2010;90:799-829.

26. Aubier M, Murciano D, Menu Y, Boczkowski J, Mal H, Pariente R. Dopamine effects on diaphragmatic strength during acute respiratory failure in chronic obstructive pulmonary disease. Ann Intern Med 1989:110:17-23.

27. Aubier M, de Troyer A, Sampson M, Macklem PT, Roussos C. Aminophylline improves diaphragmatic contractility. N Engl J Med 1981; 305:249-252.

28. Brophy C, Mier A, Moxham J, Green M. The effect of aminophylline on respiratory and limb muscle contractility in man. Eur Respir J 1989;2: 652-655.

29. Antila S, Sundberg S, Lehtonen LA. Clinical pharmacology of levosimendan. Clin Pharmacokinet 2007;46:535-552.

30. Millar NC, Homsher E. The effect of phosphate and calcium on force generation in glycerinated rabbit skeletal muscle fibers: a steadystate and transient kinetic study. J Biol Chem 1990;265:2023420240.

31. Kaheinen P, Pollesello P, Levijoki J, Haikala H. Effects of levosimendan and milrinone on oxygen consumption in isolated guinea-pig heart. J Cardiovasc Pharmacol 2004;43:555-561.

32. Vassilakopoulos T, Zakynthinos S, Roussos C. The tension-time index and the frequency/tidal volume ratio are the major pathophysiologic determinants of weaning failure and success. Am J Respir Crit Care Med 1998;158:378-385.

33. Tikunov B, Levine S, Mancini D. Chronic congestive heart failure elicits adaptations of endurance exercise in diaphragmatic muscle. Circulation 1997:95:910-916.

34. Man WD, Moxham J, Polkey MI. Magnetic stimulation for the measurement of respiratory and skeletal muscle function. Eur Respir J 2004; 24:846-860.

35. Wragg S, Hamnegard C, Road J, Kyroussis D, Moran J, Green M, Moxham J. Potentiation of diaphragmatic twitch after voluntary contraction in normal subjects. Thorax 1994;49:1234-1237.

36. Sinderby C, Spahija J, Beck J. Changes in respiratory effort sensation over time are linked to the frequency content of diaphragm electrical activity. Am J Respir Crit Care Med 2001;163:905-910.

37. Sinderby CA, Lindstrom L, Grassino AE. Automatic assessment of electromyogram quality. J Appl Physiol 1995;79:1803-1815.

38. Sinderby CA, Beck JC, Lindstrom LH, Grassino AE. Enhancement of signal quality in esophageal recordings of diaphragm EMG. $J$ Appl Physiol 1997;82:1370-1377.

39. Lilleberg JM, Sundberg S, Hayha M, Akkila J, Nieminen MS. Haemodynamic dose-efficacy of levosimendan in healthy volunteers. Eur J Clin Pharmacol 1994;47:267-274. 